\title{
Spectroscopic signatures of extratidal stars around the globular clusters NGC 6656 (M 22), NGC 3201, and NGC 1851 from RAVE
}

\author{
A. Kunder ${ }^{1}$, G. Bono ${ }^{2,3}$, T. Piffl ${ }^{4}$, M. Steinmetz ${ }^{1}$, E. K. Grebel ${ }^{5}$, B. Anguiano ${ }^{6,7}$, K. Freeman ${ }^{8}$, G. Kordopatis ${ }^{9}$, \\ T. Zwitter ${ }^{10}$, R. Scholz ${ }^{1}$, B. K. Gibson ${ }^{11,12}$, J. Bland-Hawthorn ${ }^{13}$, G. Seabroke ${ }^{14}$, C. Boeche ${ }^{5}$, A. Siebert ${ }^{15}$, \\ R. F. G. Wyse ${ }^{16}$, O. Bienaymé ${ }^{15}$, J. Navarro ${ }^{17}$, A. Siviero ${ }^{18}$, I. Minchev ${ }^{1}$, Q. Parker ${ }^{6,7,19}$, W. Reid ${ }^{6,7}$, \\ G. Gilmore ${ }^{9}$, U. Munari ${ }^{20}$, and A. Helmi ${ }^{21}$ \\ ${ }^{1}$ Leibniz-Institut für Astrophysics (AIP), An der Sternwarte 16, 14482 Potsdam Germany \\ e-mail: akunder@aip.de \\ 2 Dipartimento di Fisica, Universita di Roma Tor Vergata, Rome, Italy \\ 3 INAF-Osservatorio Astronomico di Roma, via Frascati 3300040 Monte Porzio Catone, Italy \\ 4 Rudolf Peierls Centre for Theoretical Physics, 1 Keble Road, Oxford, OX1 3NP, UK \\ 5 Astronomisches Rechen-Institut, Zentrum für Astronomie der Universität Heidelberg, Mönchhofstr. 12-14, 69120 Heidelberg, \\ Germany \\ 6 Department of Physics \& Astronomy, Macquarie University, Sydney, NSW 2109, Australia \\ 7 Research Centre for Astronomy, Astrophysics and Astrophotonics, Macquarie University, Sydney, NSW 2109, Australia \\ ${ }^{8}$ Research School of Astronomy \& Astrophysics, The Australian National University, Canberra, Australia \\ 9 Institute of Astronomy, Cambridge University, Madingley Road, Cambridge CB3 OHA, UK \\ 10 University of Ljubljana, Faculty of Mathematics and Physics, Jadranska 19, 1000 Ljubljana, Slovenia \\ 11 Institute for Computational Astrophysics, Dept of Astronomy \& Physics, Saint Mary University, Halifax, NS, BH3 3C3, Canada \\ 12 Jeremiah Horrocks Institute, University of Central Lancashire, Preston, PR1 2HE, UK \\ 13 Sydney Institute for Astronomy, School of Physics A28, University of Sydney, NSW 2006, Australia \\ 14 Mullard Space Science Laboratory, University College London, Holmbury St Mary, Dorking, RH5 6NT, UK \\ 15 Observatoire Astronomique de Strasbourg, Université de Strasbourg, CNRS, UMR 7550, 11 rue de l'Université, 67000 Strasbourg, \\ France \\ 16 Department of Physics and Astronomy, Johns Hopkins University, 3400 North Charles Street, Baltimore, MD 21218, USA \\ 17 Senior CIfAR Fellow, Department of Physics and Astronomy, University of Victoria, Victoria, BC V8P 5C2, Canada \\ 18 Department of Physics and Astronomy, Padova University, Vicolo dell'Osservatorio 2, 35122 Padova, Italy \\ 19 Australian Astronomical Observatory, PO Box 915, North Ryde, NSW 1670, Australia \\ 20 INAF National Institute of Astrophysics, Astronomical Observatory of Padova, 36012 Asiago (VI), Italy \\ 21 Kapteyn Astronomical Institute, University of Groningen, PO Box 800, 9700 AV Groningen, The Netherlands
}

Received 2 May 2014 / Accepted 25 August 2014

\section{ABSTRACT}

\begin{abstract}
Context. Stellar population studies of globular clusters have suggested that the brightest clusters in the Galaxy might actually be the remnant nuclei of dwarf spheroidal galaxies. If the present Galactic globular clusters formed within larger stellar systems, they are likely to be surrounded by extratidal halos and/or tails made up of stars that were tidally stripped from their parent systems.

Aims. The stellar surroundings around globular clusters are therefore one of the best places to look for the remnants of an ancient dwarf galaxy. Here an attempt is made to search for tidal debris around the supernovae enriched globular clusters M 22 and NGC 1851, as well as the kinematically unique cluster NGC 3201.

Methods. The stellar parameters from the RAdial Velocity Experiment (RAVE) are used to identify stars with the RAVE metallicities, radial velocities, and elemental abundances that are consistent with the abundance patterns and properties of the stars in M 22 , NGC 1851, and NGC 3201.

Results. Discovery of RAVE stars that may be associated with M 22 and NGC 1851 are reported, some of which are at projected distances $\sim 10$ degrees away from the core of these clusters. Numerous RAVE stars associated with NGC 3201 suggest that either the tidal radius of this cluster is underestimated or that there are some unbound stars extending a few arc minutes from the edge of the cluster's radius. No other extratidal stars associated with NGC 3201 could be identified. The bright magnitudes of the RAVE stars make them easy targets for high-resolution follow-up observations, eventually allowing further chemical tagging to solidify (or exclude) stars outside the tidal radius of the cluster as tidal debris. In both our radial velocity histograms of the regions surrounding NGC 1851 and NGC 3201, a peak of stars at $\sim 230 \mathrm{~km} \mathrm{~s}^{-1}$ is seen, consistent with extended tidal debris from $\omega$ Centauri.
\end{abstract}

Key words. globular clusters: individual: M 22 - Galaxy: formation - Galaxy: kinematics and dynamics - Galaxy: structure Galaxy: stellar content - Galaxy: evolution

\section{Introduction}

The classical Searle \& Zinn (1978) scenario of the formation of the Milky Way predicts that the halo globular clusters
(GCs) formed in larger dwarf galaxies. Upon the accretion of smaller galaxies, dark matter, stars, gas, and in some cases, GCs (Da Costa \& Armandroff 1995; Peñarrubia et al. 2009) will remain in the halo of the more massive galaxy. They will then 
add to any pre-existing GC population (Searle \& Zinn 1978; Zinnecker et al. 1988; Freeman 1993; Abadi et al. 2006; Forbes \& Bridges 2010; Leaman et al. 2013).

There is now good evidence that two of the most luminous GCs in the Milky Way are the nuclei of dwarf galaxies (e.g., $\omega$ Centauri, Lee et al. 1999, and M54 is associated with the Sagittarius dwarf spheroidal galaxy, Sarajedini \& Layden 2008). Also, in the past two decades, observational studies have shown the presence of tidal tails around GCs (e.g., Grillmair et al. 1995, 1996; Holland et al. 1997; Lehmann \& Scholz 1997; Leon et al. 1999, 2000; Testa et al. 2000; Odenkirchen et al. 2001, 2003; Sohn et al. 2003; Lee et al. 2003, 2004; Jordi \& Grebel 2010). Some of these tidal tails show structures that are elongated for $\sim 45$ degrees on the sky (e.g., NGC 5466 Belokurov et al. 2006; Grillmair \& Dionatos 2006) and 22 degrees on the sky, (e.g., Palomar 5, Grillmair \& Dionatos 2006). Several other stellar debris features associated with GCs have also been identified, some with a possible origin from known GCs (e.g., NGC 288, Grillmair et al. 2013) and some associated with unknown clusters (Grillmair 2009, 2011; Williams et al. 2011; Wylie-de Boer et al. 2012), awaiting additional and/or deeper imaging and spectroscopy to confirm such an association.

There is evidence that Galactic globulars can be split into two subsamples, the ones that formed in situ and those that have been accreted. The exact fraction is still under debate and depends on the criteria adopted to constrain the kinematics (e.g., Salaris \& Weiss 2002; Marín-Franch et al. 2009; VandenBerg et al. 2013; Leaman et al. 2013). The two subsamples cover a broad range in metal abundance and in total mass. This means that the latter parameter does not allow us to distinguish between the two subgroups. More recent findings suggest that the in situ GCs and the accreted ones do obey two different age-metallicity relations (VandenBerg et al. 2013). This evidence, once confirmed by independent analysis, might provide the opportunity to determine the origin of GCs. These authors suggest that the in situ clusters are, at fixed age, $\sim 0.5$ dex more metal-rich.

Mackey et al. (2004) utilized the horizontal branch (HB) morphology of a large sample of Milky Way GCs, together with GCs in dwarf spheroidal galaxies, to propose that accretion onto the early Milky Way was substantial, with 41 (27 per cent) of the GCs in the Milky Way being accreted. Using blue halo stars, Unavane et al. (1996) suggested an accretion fraction closer to ten per cent. More recently, Forbes \& Bridges (2010) have suggested that 27 to $47 \mathrm{GCs}(\sim 1 / 4$ of the entire system), from six to eight dwarf galaxies, were accreted to build the Milky Way GC system we see today. The age-metallicity relation (AMR) of Milky Way GCs furthermore seems to prove that the metal-poor GCs were formed in relatively low-mass (dwarf) galaxies and later accreted by the MW (Leaman et al. 2013), although this is still not a settled issue (see e.g., Marín-Franch et al. 2009).

Whereas tidal tails originating from a cluster could indicate that the cluster is the remnant of an ancient galaxy progressively disrupted by the interaction with the Milky Way potential, internal processes including stellar evolution, gas expulsion, and two-body relaxation (e.g., Geyer \& Burkert 2001; Kroupa 2001) can also dissolve a GC. For example, Gnedin \& Ostriker (1997) show how disk shocking affects each star in the cluster as it crosses the disk, leading to the result that stars close to the tidal boundary can be lost during disk crossing events given that this extra energy allows them to escape from the star cluster. Simulations show that tidal features are expected also for instance, for GCs on eccentric orbits near their apogalacticon (Montuori et al. 2007). Gnedin \& Ostriker (1997) find that more than half of the present Galactic GCs are to be destroyed within the next Hubble time. One might expect some difference in the stellar populations (for instance, age, elemental abundances) in the tails and main body if the cluster were indeed a remnant of a larger system in which there were gradients, although the extent may need to be considered on a case-by-case basis.

It is now accepted that most, if not all, Milky Way GCs have had at least two epochs of star formation (e.g., see the reviews by Gratton et al. 2004, 2012a). A first epoch of star formation gave origin to the "normal" (first-generation) stars, with $\mathrm{CNO}$ and other abundances similar to Population II field stars of the same metallicity. Afterward, some other epoch of star formation (second-generation) occurred, including material heavily processed through the $\mathrm{CNO}$ cycle. Hence, dissolved massive GCs can be traced by the chemical signature of their secondgeneration stars, which tend to be enhanced in light elements. Using such light element abundance anomalies in present-day halo field stars, Martell \& Grebel (2010) and Martell et al. (2011) suggest that at least $17 \%$ of the present-day stellar mass of the Milky Way halo originally formed in GCs.

GC streams can be some of the coldest stellar substructures yet discovered (e.g., Combes et al. 1999; Odenkirchen et al. 2009; Willett et al. 2009), especially compared to early accretion/dissolution events which would result in low surface brightness, dynamically hotter and spatially extended substructures. Large samples of such streams can therefore provide the possibility to map the distribution of Galactic dark matter with much greater spatial resolution than what is presently possible (e.g., Murali \& Dubinski 1999). Especially large field studies observed in the framework of the Sloan Digitial Sky Survey (SDSS; York et al. 2000) and Wide-Field Infrared Survey Explorer (WISE; Wright et al. 2010), for example, have led to an extensive mapping and detection of GC tidal tails. The RAdial Velocity Experiment (RAVE, Steinmetz et al. 2006) is one of the few kinematic surveys that has also made discoveries of stellar streams associated with GCs (e.g., Williams et al. 2011; Wyliede Boer et al. 2012). To date, RAVE has gathered a half-a-million medium-resolution spectra $(R \sim 7500)$ of stars in the Southern sky, and because RAVE's input catalog is magnitude limited $(8<I<13)$, the stellar sample is essentially homogeneous and free of kinematic biases. The cataloged stars lie mostly within $2.5 \mathrm{kpc}$ of the Sun (e.g., Burnett et al. 2011; Binney et al. 2014), although stars also belonging to the Large Magellanic Cloud at a distance of $\sim 50 \mathrm{kpc}$ have been identified within RAVE (Munari et al. 2009).

In this paper, the first results from a search for extratidal stars around the halo GCs M 22, NGC 1851 and NGC 3201 in the RAVE survey footprint are presented. These clusters were chosen for a number of reasons. First, unlike a number of other clusters within the RAVE footprint, they were not specifically targeted by RAVE, so the relative frequency of stars identified within the cluster tidal radius and outside the cluster tidal radius can be directly compared. Second, these clusters are already good candidates for having an extragalactic origin. Third, these clusters have radial velocities and/or $[\mathrm{Fe} / \mathrm{H}]$ abundances that are largely offset from disk field stars seen in projection along that line of sight, reducing field star contamination issues. Lastly, a number of stars within these clusters have already been wellstudied spectroscopically, so the light elements of potential extratidal candidates can provide important clues as to their cluster association. The basic properties and distances of these clusters are listed in Table 1.

We define an extratidal star to be an unbound star when it exceeds the tidal radius (e.g., Meylan \& Heggie 1997), as $N$-body models show that this leads to consistent results 
Table 1. Globular cluster sample.

\begin{tabular}{lccccccccccc}
\hline \hline Cluster name & $\begin{array}{c}\text { RA } \\
(\mathrm{J} 2000)\end{array}$ & $\begin{array}{c}\text { Dec } \\
(\mathrm{J} 2000)\end{array}$ & $\begin{array}{c}l \\
\left(^{\circ}\right)\end{array}$ & $\begin{array}{c}b \\
\left({ }^{\circ}\right)\end{array}$ & $\begin{array}{c}R_{\odot} \\
(\mathrm{kpc})\end{array}$ & $\begin{array}{c}R_{G C} \\
(\mathrm{kpc})\end{array}$ & $\begin{array}{c}{[\mathrm{Fe} / \mathrm{H}]} \\
(\mathrm{dex})\end{array}$ & $\begin{array}{c}V_{\mathrm{LOS}} \\
\left(\mathrm{km} \mathrm{s}^{-1}\right)\end{array}$ & $\begin{array}{c}r_{c} \\
(')\end{array}$ & $\begin{array}{c}\text { King } r_{t} \\
(')\end{array}$ & $\begin{array}{c}\text { Wilson } r_{t} \\
(')\end{array}$ \\
\hline NGC 3201 & 101736.82 & -462444.9 & 277.23 & 8.64 & 4.9 & 8.8 & -1.59 & 494.0 & 1.30 & 25.35 & 54.8 \\
NGC 1851 & 051406.76 & -4020247.6 & 244.51 & -35.03 & 12.1 & 16.6 & -1.18 & 320.5 & 0.09 & 6.52 & 44.7 \\
NGC 6656 (M 22) & 183623.94 & -235417.1 & 9.89 & -7.55 & 3.2 & 4.9 & -1.70 & -146.3 & 1.33 & 31.90 & 119.9 \\
\hline
\end{tabular}

Table 2. Globular cluster member selection criteria.

\begin{tabular}{ll}
\hline \hline Cluster name & Selection criteria \\
\hline NGC 3201 & $480 \mathrm{~km} \mathrm{~s}^{-1}<\mathrm{RV}<510 \mathrm{~km} \mathrm{~s}^{-1} ; \mathrm{RV}$ error $<10 \mathrm{~km} \mathrm{~s}^{-1}$ \\
NGC 1851 & $300 \mathrm{~km} \mathrm{~s}^{-1}<\mathrm{RV}<380 \mathrm{~km} \mathrm{~s}^{-1} ; \mathrm{RV}$ error $<10 \mathrm{~km} \mathrm{~s}^{-1} ; K>-7 \times(J-K)+14$ \\
NGC 6656 (M 22) & $-200 \mathrm{~km} \mathrm{~s}^{-1}<\mathrm{RV}<-120 \mathrm{~km} \mathrm{~s}^{-1} ;[\mathrm{Fe} / \mathrm{H}]<-1.25 \mathrm{dex} ; S / N>21 ;$ AlgoConv $=0$ \\
\hline
\end{tabular}

(Giersz \& Heggie 1997). RAVE red giant candidates in M22, NGC 1851 and NGC 3201, are presented, which lie outside the cluster tidal radius, but which were still likely born in these clusters as evidenced by their radial velocities, metallicities, and elemental abundances.

\section{Detection in RAVE}

The fourth data release (DR4) of RAVE contains stellar atmospheric parameters (effective temperature, surface gravity, overall metallicity), radial velocities, individual abundances and distances determined for 425561 stars (Kordopatis et al. 2013). This wealth of information and the extensive spatial distribution presents an ideal foundation for our study. From this sample, 187305 stars have spectra for which the chemical abundance pipeline is able to determine abundances for magnesium, aluminum, silicon, titanium, iron and nickel (Boeche et al. 2011). The uncertainties on these quantities vary as a function of signalto-noise ratio $(\mathrm{S} / \mathrm{N})$, number of lines that could be measured, and also depend on the specific element. Typically, the uncertainty in $[\mathrm{Fe} / \mathrm{H}]$ is $\sim 0.23 \mathrm{dex}$, the uncertainty for $\mathrm{Mg}, \mathrm{Al}$, and $\mathrm{Si}$ is $\sim 0.2$ dex, and the uncertainty for $\mathrm{Ti}$ and $\mathrm{Ni}$ is $\sim 0.3 \mathrm{dex}$ (see discussion and Figs. 13-17 in Kordopatis et al. 2013, for further details). Because the GCs NGC 3201 and NGC 1851 have stars with radial velocities offset from the rest of the field population by at least one hundred $\mathrm{km} \mathrm{s}^{-1}$, stars belonging to these clusters can be provisionally identified from their radial velocities, colors and magnitudes alone.

Whenever possible, RAVE stellar abundances are used to further link extratidal stars to a cluster, although the number of RAVE spectra with reliable abundances is not nearly as extensive ( $\sim 1 / 4$ the sample) as the RAVE sample of stars with welldetermined radial velocities. However, it is not always obvious that the metallicity of an extratidal star should be the same as a cluster star, for example, if the metallicities of extratidal stars should be the same between the GC and its dwarf galaxy host. For instance, if a closed-box model of chemical evolution is assumed, and assuming that the yield of each generation of stars is constant, then the variation of metallicity $(Z)$ with time can be expressed as

$Z(t)=-\rho \ln \frac{M_{\mathrm{g}}(t)}{M_{\mathrm{g}}(0)}$

(Binney \& Merrifield 1998). Here, $\left[M_{\mathrm{g}}(t) / M_{\mathrm{g}}(0)\right]$ is the ratio of gas masses, and is unity at $t=0$ and zero at the present time ( $t=14 \mathrm{Gyr}$ ). The yield is given by $\rho$. Setting $\rho$ to 0.005 so as to match the zero point of the Sagittarius dwarf spheroidal galaxy
(Sgr) age-metallicity relation, of which the GC M54 is thought to be the nucleus (Layden \& Sarajedini 2000), then $[\mathrm{Fe} / \mathrm{H}]$ would become more metal-rich by $\sim 0.5$ dex with every $\sim 4$ Gyr that passed. Hence, if we accept the possibility that a nucleated dwarf galaxy, with a Milky Way GC as its nucleus, formed stars continuously in relative isolation with little or no infall or release of its interstellar gas, and that the yield is similar to that of Sgr, then we would not expect a large difference in $[\mathrm{Fe} / \mathrm{H}]$ between the GC and the extratidal stars, unless we are probing extratidal stars with a multiple Gyr age difference from the GC.

It is important to point out, that although a closed-box model of chemical evolution fits the age-metallicity relation of Sgr galaxy as a whole well (Layden \& Sarajedini 2000), this is not the case for the formation and evolution of the anomalous GC $\omega$ Cen (e.g., Romano et al. 2006). However, the $[\mathrm{Fe} / \mathrm{H}]$ spread of the stars within $\omega$ Cen is still thought to be similar to those in its extended tidal debris (Majewski et al. 2012). Ultimately, it may be that the exact nature of the metallicity of an extratidal star linked to a host dwarf galaxy and the metallicity of a star in its nucleus (now observed as a Milky Way GC) depends on the particular cluster/system under study and must be considered on a case-by-case basis.

We also investigated using proper motions of the RAVE stars as an exclusion criterion in the selection of extratidal stars, but the errors of available measurements prevented the use of proper motions at this stage. The properties of the GCs studied here are listed in Table 1, taken from Harris (2010 edition of 1996), except for the Wilson (1975) tidal radius, which is from McLaughlin \& van der Marel (2005). The columns contain (1) the cluster name; (2) the right ascension in hours, minutes and seconds (epoch J2000); (3) the declination in degrees, arcminutes and arcseconds; (4) the Galactic longitude in degrees; (5) the Galactic latitude in degrees; (6) the distance from Sun in kiloparsecs $(\mathrm{kpc})$; (7) the distance from Galactic center $(\mathrm{kpc})$, assuming $R_{0}=8.0 \mathrm{kpc}$ (e.g., Groenewegen et al. 2008; Matsunaga et al. 2009); (8) the [Fe/H] metallicity; (9) the Heliocentric radial velocity in $\mathrm{km} \mathrm{s}^{-1}$; (10) the core radius in arcmin; (11) the King (1966) tidal radius in arcmin; and (11) the Wilson (1975) tidal radius in arcmin from McLaughlin \& van der Marel (2005).

The specific selection criteria to search for cluster stars belonging to these GCs are listed in Table 2. Briefly, for NGC 3201, we only select candidates based on their radial velocity and the errors in radial velocity measurements. For the cluster NGC 1851, a color criterion is also used, and for M 22, only stars with derived metallicities from RAVE are used. Although ideally we would prefer to select cluster stars based on their radial 

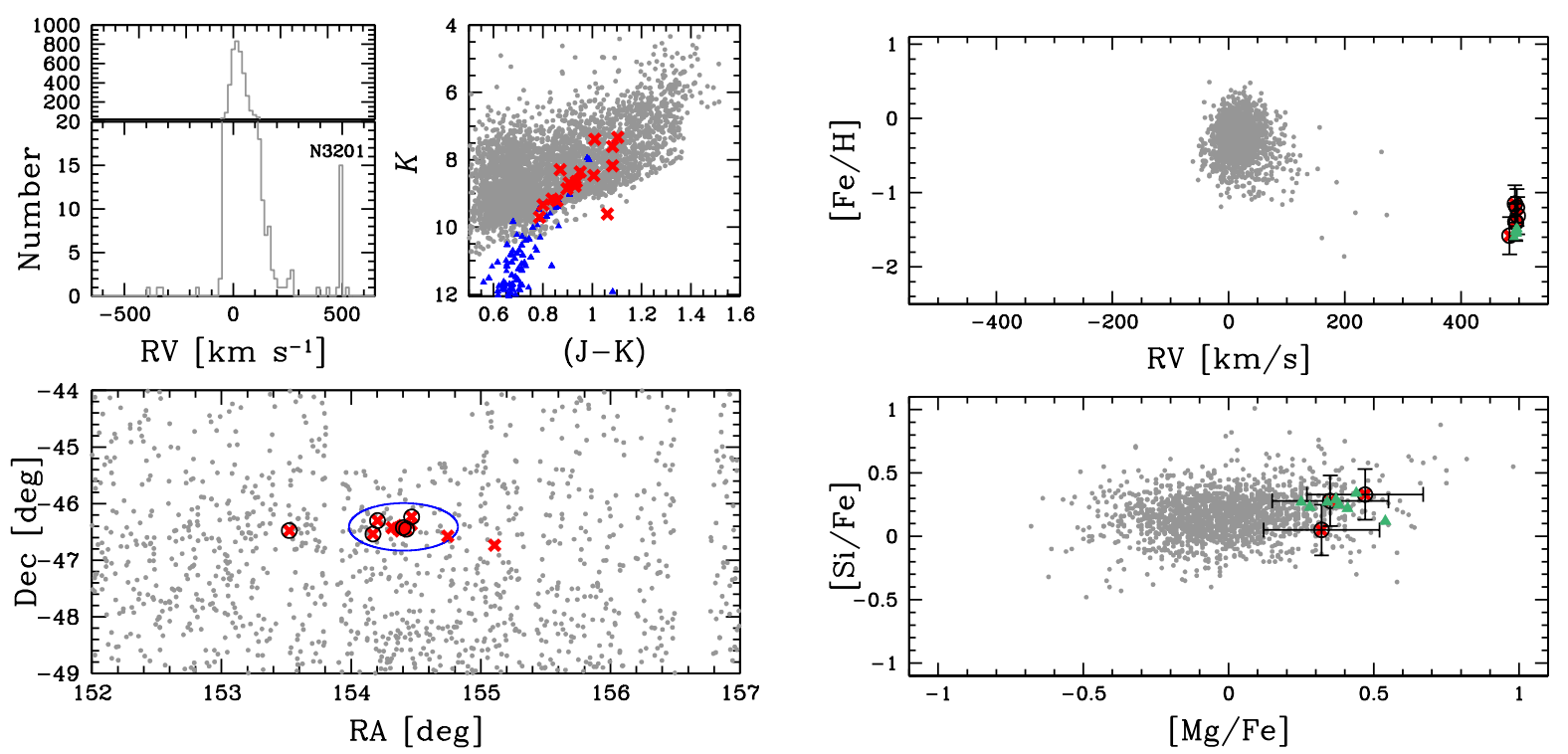

Fig. 1. Left: CMD (top-right), and radial velocity distribution (top-left) of the RAVE stars in a 12 degree field centered on NGC 3201 . The spatial distribution of the RAVE stars in a 5 degree field centered on the cluster is shown (bottom), and the King tidal radius of the cluster is shown by the large circle. The crosses (red in online version) show the RAVE stars whereas the triangles (blue in the online version) show the NGC 3201 stars studied by Côté et al. (1994). The circled RAVE stars are those for which a chemical analysis could be carried out. Right: [Fe/H], [Si/Fe] and $[\mathrm{Mg} / \mathrm{Fe}]$ ratio of a subsample of these same stars for which abundances could be determined. The triangles (green in the online version) are the parameters of the NGC 3201 red giant stars obtained from intermediate-resolution spectra by Carretta et al. (2009) and high-resolution spectra by Muñoz et al. (2013).

velocity alone, this is only realistic for NGC 3201, the cluster with the most extreme velocity. The AlgoConv criteria indicates which stars have reliable abundances, but as mentioned previously, greatly restricts the sample of RAVE stars. Therefore, this criteria is only adopted for the cluster M 22, the cluster with the least extreme radial velocity and for which large reddening effects makes selecting cluster stars by color difficult.

\subsection{NGC 3201}

NGC 3201 has long been known as a kinematically unique GC: It has the most extreme radial velocity of the Milky Way GCs, with a heliocentric radial velocity of $494 \mathrm{~km} \mathrm{~s}^{-1}$ (2010 edition of Harris 1996). It also has a large azimuthal velocity of $250 \mathrm{~km} \mathrm{~s}^{-1}$ around the Galactic center (Gonzalez \& Wallerstein 1998), but in a retrograde sense. These kinematic peculiarities have been taken as strong evidence of a possible extragalactic formation with subsequent capture by the Milky Way (e.g., Rodgers \& Paltoglou 1984; van den Bergh 1993).

High-resolution spectroscopy carried out on the individual stars in NGC 3201 find that despite its extreme kinematics, NGC 3201 shows no large spread in $[\mathrm{Fe} / \mathrm{H}]$ compared to the observational errors nor large chemical differences with respect to other Milky Way GCs (e.g., Gonzalez \& Wallerstein 1998; Carretta et al. 2009; Muñoz et al. 2013, although see Simmerer et al. 2013). Therefore, its origin appears to be similar to "normal" GCs, for example, with signs of multiple populations, but without signs of supernova enrichment.

Figure 1 (left) shows the spatial location of NGC 3201 within the RAVE footprint. A 5 degree field of view centered on the cluster is shown. We search for cluster members based solely on the radial velocity information provided by RAVE. A radial velocity range of $\pm 15 \mathrm{~km} \mathrm{~s}^{-1}$ is searched, as this velocity range encompasses almost all stars in the extensive NGC 3201 radial velocity catalog of Côté et al. (1994, 1995). Out of the 4390 RAVE stars within a 12 degree area of the sky, 16 have a radial velocities consistent with cluster membership (i.e., radial velocities between $480 \mathrm{~km} \mathrm{~s}^{-1}$ and $510 \mathrm{~km} \mathrm{~s}^{-1}$ ). Most of these stars stars fall within the tidal radius of the cluster, but two do not (see Fig. 1). These possible extratidal stars are given in Table 3, where the columns list (1) the RAVE ID; (2) the right ascension in degrees (epoch J2000); (3) the declination in degrees; (4) the line of sight radial velocity in $\mathrm{km} \mathrm{s}^{-1}$; (5) the RAVE radial velocity uncertainty in $\mathrm{km} \mathrm{s}^{-1}$; (6) the RAVE $[\mathrm{Fe} / \mathrm{H}]$; (7) the RAVE [Si/Fe]; (8) the RAVE [Mg/Fe]; (9) the 2MASS $J$-magnitude; (10) the 2MASS $K$-magnitude; and (11) the distance from the cluster center in degrees. Increasing the area of the sky to cover 20 degrees of the RAVE footprint increases the number of RAVE stars sampled to 8495, but no additional stars with velocities of $\sim 494 \mathrm{~km} \mathrm{~s}^{-1}$ are detected. In general, the stars with velocities of $\sim 494 \mathrm{~km} \mathrm{~s}^{-1}$ also have colors and magnitudes that place them on the giant branch of NGC 3201, additionally indicating their association with the cluster. The RAVE stellar parameters are consistent with these stars being giant stars, as they all fall within the temperature and gravity range of $4000 \mathrm{~K}<$ $T_{\text {eff }}<7000 \mathrm{~K}$ and $\log g<3.5$ (cgs), clearly separating them from nearby dwarf stars.

Six of the NGC 3201 stars have metallicities derived from the RAVE chemical abundance pipeline and are shown in Fig. 1 (right). Given the external errors $(\sim 0.23$ dex $)$ in the RAVE $[\mathrm{Fe} / \mathrm{H}]$ metallicities as discussed previously, we do not wish to place too much emphasis on the metallicites to characterize the potential extratidal stars. However, it is worth stressing that the low metallicities of these stars set them apart from the majority of the RAVE stars and confirm their halo membership. Moreover, three of these stars have spectra for which elemental abundances can be determined, all of which show enhanced $[\mathrm{Si} / \mathrm{Fe}]$ and $[\mathrm{Mg} / \mathrm{Fe}]$ values, in good agreement with high-resolution spectroscopy of NGC 3201 from Carretta et al. (2009) and Muñoz et al. (2013). 
Table 3. RAVE NGC 3201 extratidal stars.

\begin{tabular}{lcccccccccc}
\hline \hline RAVE ID & $\begin{array}{c}\text { RA } \\
(\mathrm{J} 2000)\end{array}$ & $\begin{array}{c}\text { Dec } \\
(\mathrm{J} 2000)\end{array}$ & $\begin{array}{c}V_{\mathrm{LOS}} \\
\left(\mathrm{km} \mathrm{s}^{-1}\right)\end{array}$ & $\begin{array}{c}\sigma_{V} \\
\left(\mathrm{~km} \mathrm{~s}^{-1}\right)\end{array}$ & $\begin{array}{c}{[\mathrm{Fe} / \mathrm{H}]} \\
(\mathrm{dex})\end{array}$ & $\begin{array}{r}{[\mathrm{Si} / \mathrm{Fe}]} \\
(\mathrm{dex})\end{array}$ & $\begin{array}{c}{[\mathrm{Mg} / \mathrm{Fe}]} \\
(\mathrm{dex})\end{array}$ & $\begin{array}{c}J \\
(\mathrm{mag})\end{array}$ & $\begin{array}{c}K \\
(\mathrm{mag})\end{array}$ & $\begin{array}{c}r \\
(\mathrm{degrees})\end{array}$ \\
\hline 20110520_1023m47_056 & 153.5235 & -46.4779 & 495 & 1 & -1.41 & 0.05 & 0.32 & $10.01 \pm 0.02$ & $9.17 \pm 0.02$ & 0.88 \\
20090404_1023m47_058 & 155.1078 & -46.7349 & 497 & 1 & -1.24 & - & - & $8.41 \pm 0.03$ & $7.40 \pm 0.02$ & 0.78 \\
\hline
\end{tabular}
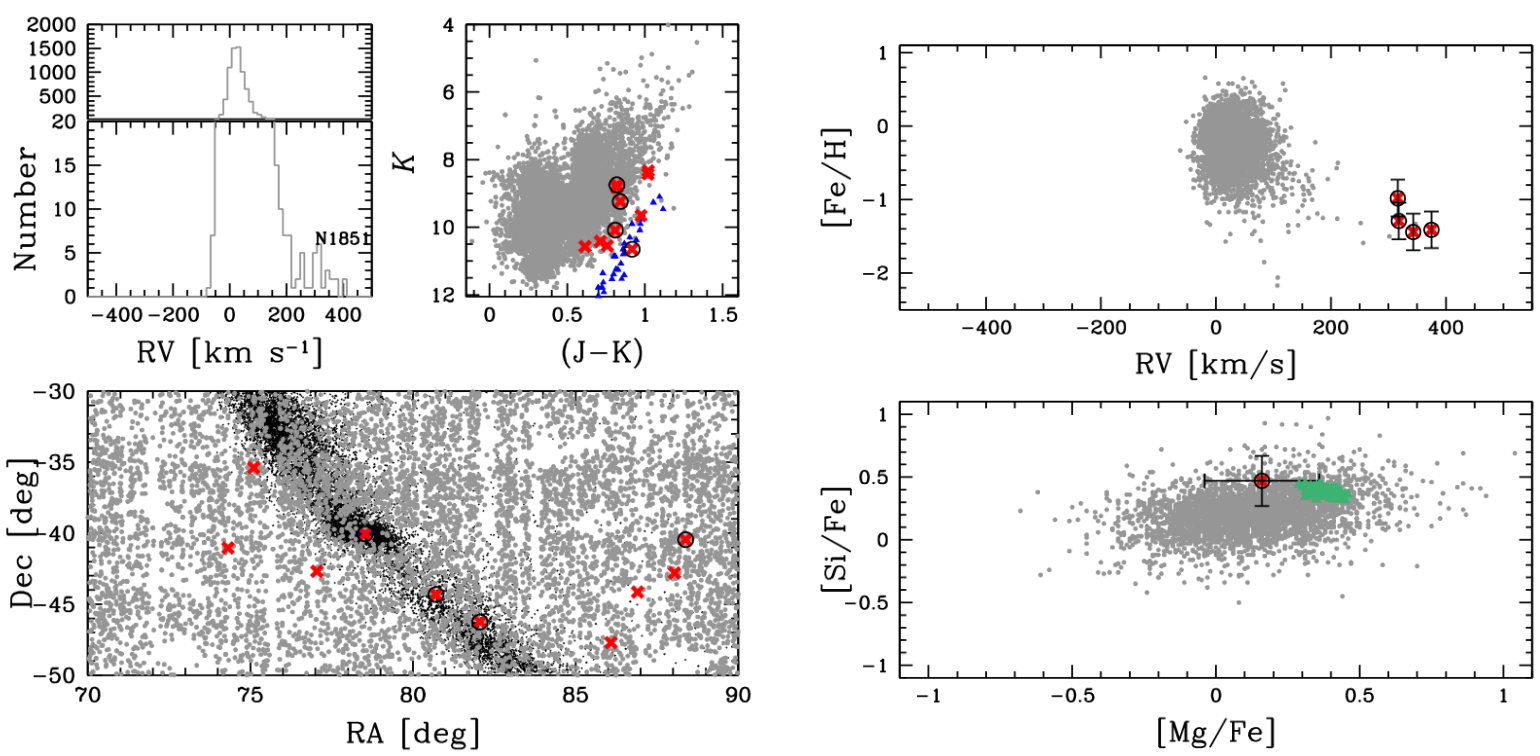

Fig. 2. Left: spatial distribution (bottom), CMD (top-right), and radial velocity distribution of the RAVE stars in a 20 degree field centered on NGC 1851. The crosses (red in the online version) show the RAVE stars whereas the triangles (blue in the online version) show the NGC 1851 stars from Milone et al. (2009) and Carretta et al. (2011). The circled RAVE stars are those for which a chemical analysis could be carried out. In the bottom panel we also show the expected angular distribution of tidal debris stars (black dots) as predicted by an $N$-body simulation of the cluster orbiting the MW for one Gyr on the most likely orbit (see Sect. 3.2 for details). Right: $[\mathrm{Fe} / \mathrm{H}],[\mathrm{Si} / \mathrm{Fe}]$ and $[\mathrm{Mg} / \mathrm{Fe}] \mathrm{ratio}$ of a subsample of these same stars for which abundances could be determined. The triangles (green in the online version) are the parameters of the NGC 1851 red giant stars obtained from intermediate-resolution spectra by Carretta et al. $(2011,2012)$.

\subsection{NGC 1851}

It is traditionally considered that "normal" GCs are those that exhibit the following properties: (i) their stars appear to have the same $[\mathrm{Fe} / \mathrm{H}]$; (ii) their stars are chemically homogeneous in the heavy elements; and (iii) their stars are chemically inhomogeneous in the light element abundances, e.g., variations in the $\mathrm{CH}, \mathrm{CN}, \mathrm{NH}$ bands, and in the $\mathrm{O}, \mathrm{Na}, \mathrm{Al}$, and $\mathrm{Mg}$ abundances. Nearly all GCs have these characteristics. NGC 1851 is one of few GCs that show a spread in the heavier elements and $[\mathrm{Fe} / \mathrm{H}]$ ( $\mathrm{rms}$ scatter $\sim 0.05 \mathrm{dex}$ in $[\mathrm{Fe} / \mathrm{H}]$, which is larger than the observational errors; Carretta et al. 2011). This is a characteristic that is seen only in a small number of other systems ${ }^{1}$. The presence of heavy element abundance ranges in these systems necessarily means their nucleosynthetic history must be more complicated than for "normal" GCs, although the exact cause remains unclear. However, the chemical analogies with the cluster systems $\omega$ Centauri and M 54, usually believed to be associated with the remnants of a dwarf galaxy cannibalized by the Milky Way, suggest the intriguing idea that all of the clusters with $[\mathrm{Fe} / \mathrm{H}]$ metallicity spreads could be surviving nuclei of

1 The other GCs distinguishable from normal GCs as evidenced by their spread in $[\mathrm{Fe} / \mathrm{H}]$ are $\omega$ Centauri (Lee et al. 1999; Bedin et al. 2004), M 54 (Layden \& Sarajedini 1997; Siegel et al. 2007), Terzan 5 (Ferraro et al. 2009), M 22 (Norris \& Freeman 1983; Da Costa et al. 2009; Marino et al. 2009, 2011) and NGC 2419 (Cohen et al. 2010; Di Criscienzo et al. 2011). more massive systems. NGC 1851 is therefore a strong candidate to search for extratidal stars.

NGC 1851 further has a split subgiant branch (Milone et al. 2008), a split RGB when particular filters are used (Han et al. 2009) and spectroscopic observations have revealed a bimodality in $s$-process abundances (Yong \& Grundahl 2008; Villanova et al. 2010; Gratton et al. 2012b).

Figure 2 shows the spatial location of NGC 1851 within the RAVE survey. The central velocity dispersion is twice as large for this cluster as compared to NGC 3201 (Harris 1996), so a wider range in radial velocity is searched. We also only consider stars as potential cluster members if their magnitudes and colors are within that of the cluster (see Table 2). Here we allow for a $\sim 2 \mathrm{kpc}$ distance spread in the color magnitude diagram (CMD), which encompasses $\sim 95 \%$ of the stars in our $N$-body simulation of the cluster orbiting the MW for one Gyr on the most likely orbit (see Sect. 3.2 for details). This also encompasses the uncertainties in the 2MASS magnitudes, which can reach up to $\sim 0.17$ mag in $K$ for the stars in the cluster core (see Table 4).

Out of the 8948 RAVE stars surveyed in a 20 degree field around NGC 1851, eleven have radial velocities, colors and magnitudes consistent with that of the cluster, and these stars are listed in Table 4. Most of these stars lie outside the tidal radius of the cluster. The two RAVE stars located within the tidal radius of the cluster almost certainly are cluster members, and the nine RAVE stars which also align along the AGB and RGB of NGC 1851 are plausible cluster members, as they have properties consistent with not only the cluster's radial velocity, but also 
Table 4. RAVE NGC 1851 stars.

\begin{tabular}{|c|c|c|c|c|c|c|c|c|c|c|}
\hline RAVE ID & $\begin{array}{c}\text { RA } \\
(\mathrm{J} 2000)\end{array}$ & $\begin{array}{c}\text { Dec } \\
(\mathrm{J} 2000)\end{array}$ & $\begin{array}{c}V_{\mathrm{LOS}} \\
\left(\mathrm{km} \mathrm{s}^{-1}\right)\end{array}$ & $\begin{array}{c}\sigma_{V} \\
\left(\mathrm{~km} \mathrm{~s}^{-1}\right)\end{array}$ & $\begin{array}{c}{[\mathrm{Fe} / \mathrm{H}]} \\
(\mathrm{dex})\end{array}$ & $\begin{array}{r}{[\mathrm{Si} / \mathrm{Fe}]} \\
(\mathrm{dex})\end{array}$ & $\begin{array}{c}{[\mathrm{Mg} / \mathrm{Fe}]} \\
(\mathrm{dex})\end{array}$ & $\begin{array}{c}J \\
(\mathrm{mag})\end{array}$ & $\begin{array}{c}K \\
(\mathrm{mag})\end{array}$ & $\begin{array}{c}r \\
\text { (degrees) }\end{array}$ \\
\hline 20051110_0449m41_116 & 74.3136 & -41.0417 & 308 & 2 & - & - & - & $10.63 \pm 0.02$ & $9.65 \pm 0.02$ & 4.33 \\
\hline 20081017_0449m41_116b & 74.3136 & -41.0417 & 305 & 1 & - & - & - & $10.63 \pm 0.02$ & $9.65 \pm 0.02$ & 4.33 \\
\hline 20100124_0501m36_070 & 75.1000 & -35.3876 & 354 & 1 & - & - & - & $9.44 \pm 0.03$ & $8.42 \pm 0.02$ & 5.78 \\
\hline 20111020_0505m41_122 & 77.0456 & -42.6795 & 359 & 1 & - & - & - & $9.36 \pm 0.02$ & $8.34 \pm 0.02$ & 3.02 \\
\hline 20111020_0505m41_095 & 78.5297 & -40.0491 & 318 & 1 & -1.29 & - & - & $11.57 \pm 0.09$ & $10.65 \pm 0.11$ & 0.0030 \\
\hline 20061120_0505m41_094 ${ }^{a}$ & 78.5285 & -40.0457 & 323 & 1 & - & - & - & $9.64 \pm 0.38$ & $8.82 \pm 0.17$ & 0.0009 \\
\hline 20120107_0532m42_060 & 80.7067 & -44.3188 & 375 & 2 & -1.41 & - & - & $9.56 \pm 0.02$ & $8.74 \pm 0.02$ & 4.80 \\
\hline 20070206_0536m47_056 & 82.0560 & -46.2541 & 343 & 1 & -1.44 & - & - & $10.08 \pm 0.02$ & $9.24 \pm 0.03$ & 7.14 \\
\hline 20041101_0523m48_083 ${ }^{b}$ & 82.0560 & -46.2541 & 341 & 2 & - & - & - & $10.08 \pm 0.02$ & $9.24 \pm 0.03$ & 7.14 \\
\hline 20050331_0555m46_021 & 86.0893 & -47.7149 & 321 & 7 & - & - & - & $11.13 \pm 0.02$ & $10.42 \pm 0.02$ & 10.77 \\
\hline 20040209_0555m46_063 & 86.9080 & -44.1528 & 316 & 4 & - & - & - & $11.18 \pm 0.02$ & $10.57 \pm 0.02$ & 9.33 \\
\hline 20050221_0549m40_146 & 88.0446 & -42.8051 & 311 & 1 & - & - & - & $11.30 \pm 0.02$ & $10.54 \pm 0.02$ & 9.91 \\
\hline 20120122_0558m41_132 & 88.3831 & -40.4547 & 316 & 1 & -0.98 & 0.47 & 0.16 & $10.89 \pm 0.02$ & $10.09 \pm 0.02$ & 9.86 \\
\hline
\end{tabular}

Notes. ${ }^{(a)}$ Within tidal radius; ${ }^{(b)}$ star observed twice by RAVE.
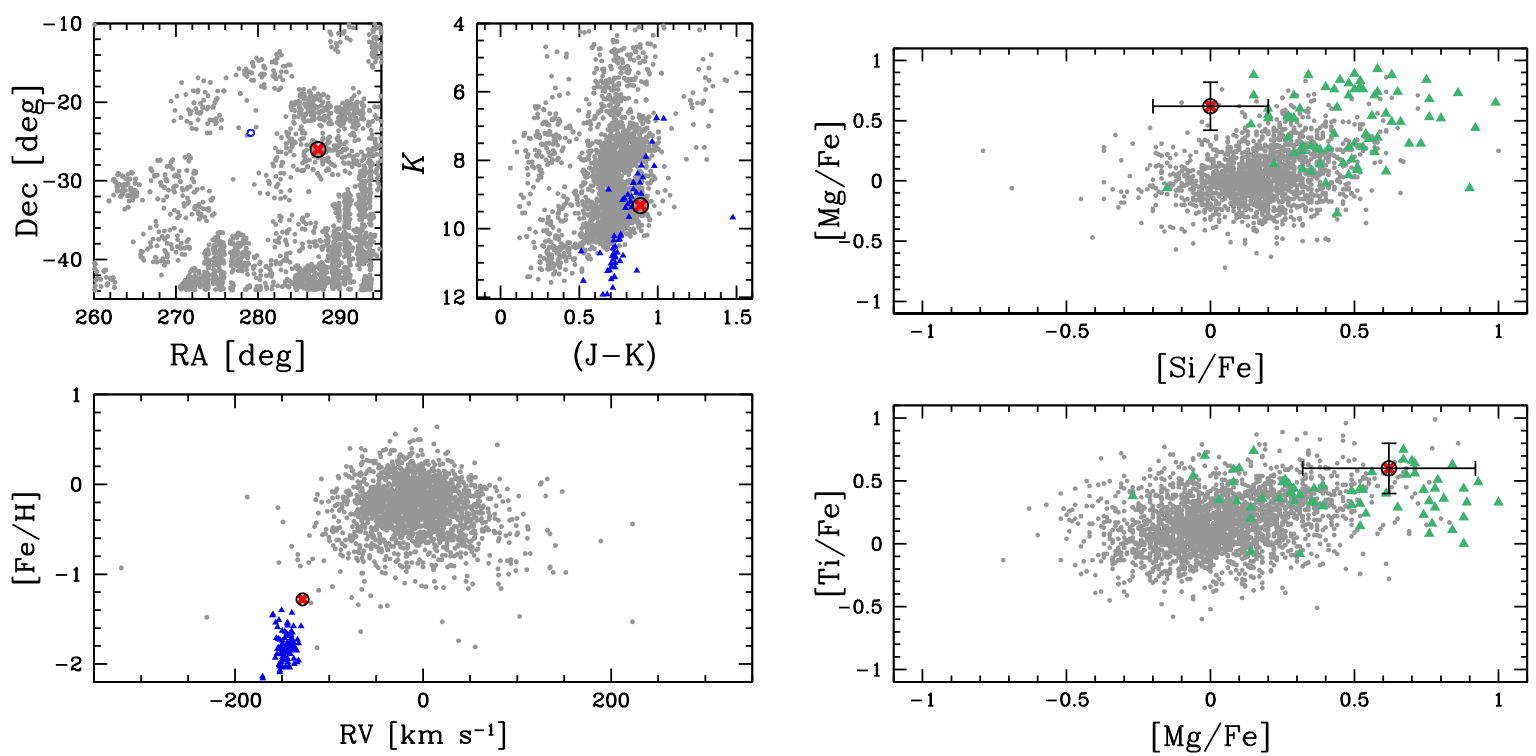

Fig. 3. Left: spatial distribution (top-left), CMD (top-right), and radial velocity vs. $[\mathrm{Fe} / \mathrm{H}]$ distribution of the RAVE stars in a 35 degree field centered on NGC 1851. The cluster center of M 22 is indicated by an open (blue) circle. The $[\mathrm{Fe} / \mathrm{H}]$, radial velocity, colors and magnitudes of the red giant branch stars of M 22 shown as triangles (blue in the online version) are taken from Marino et al. (2009), Da Costa et al. (2009), Alves-Brito et al. (2012) and Gratton et al. (2014). Right: $[\mathrm{Si} / \mathrm{Fe}],[\mathrm{Mg} / \mathrm{Fe}]$ and $[\mathrm{Ti} / \mathrm{Fe}]$ ratio of the same stars. The triangles (green in the online version) are elemental abundances of M 22 horizontal branch stars obtained from intermediate-resolution spectra by Gratton et al. (2014).

its distance. Furthermore, for the RAVE stars with a $\mathrm{S} / \mathrm{N}$ greater than 40, the RAVE temperatures and gravities are in good agreement with the stars being giant branch stars of NGC 1851 .

Figure 2 (right) shows the derived $[\mathrm{Fe} / \mathrm{H}]$ abundances for the four RAVE stars with spectra suitable for abundance determination. Although the rms spread in $[\mathrm{Fe} / \mathrm{H}]$ as determined for this cluster from high-resolution spectra is $\sim 0.05$ dex (Carretta et al. 2011), the individual errors in the RAVE metallicity values $(\sim 0.23$ dex $)$ are considerably larger than this, and so it is not surprising that the $[\mathrm{Fe} / \mathrm{H}]$ values show a broader spread. Within the errors, all the four stars with radial velocities consistent with that of NGC 1851 also have $[\mathrm{Fe} / \mathrm{H}]$ metallicities in agreement with the $[\mathrm{Fe} / \mathrm{H}]$ of the cluster and are significantly more metalpoor then the majority of the field population seen in projection along that line of sight in this area of the sky. One of these stars also has an estimate of $[\mathrm{Si} / \mathrm{H}]$, and $[\mathrm{Mg} / \mathrm{H}]$, which is consistent within its uncertainty with those of the cluster.

\subsection{NGC $6656-M 22$}

M 22, like NGC 1851, is another one of the six GCs known to host groups of stars with different iron $[\mathrm{Fe} / \mathrm{H}]$ and heavy element contents. The stellar rms scatter is $[\mathrm{Fe} / \mathrm{H}] \sim 0.15 \mathrm{dex}$ and variations in its elements associated with $s$-process elements are also present (Norris \& Freeman 1983; Da Costa et al. 2009; Marino et al. 2009, 2011). This direct evidence for extended star formation suggests this cluster may have an extragalactic origin and hence a tidal tail. The CMD of M 22 shows a double subgiant branch (Marino et al. 2012) as well as two discrete distributions of RGBs, each having a different $\mathrm{Ca}$ abundance as observed from the $h k$ index of the Ca-by photometry (Lee et al. 2009).

RAVE avoided probing close to the Galactic plane, so the number of RAVE stars is not as dense in this region of the sky as e.g., NGC 1851. Figure 3 shows the spatial location of the GC M 22, with the 2162 RAVE stars in a 35 degree field of 
Table 5. RAVE NGC 6656 extratidal stars.

\begin{tabular}{lccccccccccc}
\hline \hline RAVE ID & $\begin{array}{c}\text { RA } \\
(\mathrm{J} 2000)\end{array}$ & $\begin{array}{c}\mathrm{Dec} \\
(\mathrm{J} 2000)\end{array}$ & $\begin{array}{c}V_{\mathrm{LOS}} \\
\left(\mathrm{km} \mathrm{s}^{-1}\right)\end{array}$ & $\begin{array}{c}\sigma_{V} \\
\left(\mathrm{~km} \mathrm{~s}^{-1}\right)\end{array}$ & $\begin{array}{c}{[\mathrm{Fe} / \mathrm{H}]} \\
(\mathrm{dex})\end{array}$ & $\begin{array}{c}{[\mathrm{Si} / \mathrm{Fe}]} \\
(\mathrm{dex})\end{array}$ & $\begin{array}{c}{[\mathrm{Ti} / \mathrm{Fe}]} \\
(\mathrm{dex})\end{array}$ & $\begin{array}{c}{[\mathrm{Al} / \mathrm{Fe}]} \\
(\mathrm{dex})\end{array}$ & $\begin{array}{c}{[\mathrm{Mg} / \mathrm{Fe}]} \\
(\mathrm{dex})\end{array}$ & $\begin{array}{c}J \\
(\mathrm{mag})\end{array}$ & $\begin{array}{c}K \\
(\mathrm{mag})\end{array}$ \\
\hline $20110824 \_1915 \mathrm{~m} 27 \_053$ & 287.3046 & -26.0189 & -128 & 1 & -1.28 & 0.00 & 0.60 & 0.26 & 0.62 & $10.21 \pm 0.02$ & $9.32 \pm 0.03$ \\
\hline
\end{tabular}

view. Because this cluster does not have as extreme a velocity as for NGC 1851 and NGC 3201, the RAVE stars are restricted to only those which also have reliable abundance determinations, as indicated by sufficient $\mathrm{S} / \mathrm{N}$ and an AlgoConv value that indicates that the DR4 pipeline converged (see Kordopatis et al. 2013, for further details). Because the stars in M 22 do not have a homogenous distribution of $[\mathrm{Fe} / \mathrm{H}]$, but have been shown to range from -1.25 to -2.4 dex (e.g., Gratton et al. 2014), our $[\mathrm{Fe} / \mathrm{H}]$ search criteria cover all RAVE stars more metal poor than -1.25 dex. Figure 3 (bottom, left panel) shows the range of $[\mathrm{Fe} / \mathrm{H}]$ and radial velocity observed for stars in $\mathrm{M} 22$, which guided our search criteria for potential extratidal stars belonging to this cluster. The exact criteria are explicitly listed in Table 2.

One of these stars has a radial velocity and metallicity consistent with that of M22, and is located at a projected distance $\sim 10$ degrees from the cluster center. It also has a color and magnitude that places it on the giant branch of M22, as well as $[\mathrm{Ti} / \mathrm{Fe}],[\mathrm{Mg} / \mathrm{Fe}]$ and $[\mathrm{Si} / \mathrm{Fe}]$ values consistent with those of the cluster.

\section{Discussion}

A cluster's tidal radius is normally estimated by fitting King (1966) models to cluster density profiles. However, the assumption of the King edge radius as a real physical limit of the cluster is not set in stone. For example, McLaughlin \& van der Marel (2005) investigate the stellar distributions of GCs in not only the Galaxy, but also the Magellanic Clouds and the Fornax dwarf spheroidal galaxy, and find that Wilson (1975) models, originally developed for application to elliptical galaxies, describe the outer structures better than do King models. More recent investigations capitalizing on wide-field imagers have confirmed this result (e.g., Di Cecco et al. 2013).

Also, Zocchi et al. (2012) show with their sample of 13 GCs, that kinematic fits are crucial to assessing whether a model is actually suited to describing any given GC. Unfortunately, there is a general lack of good kinematic data, generally consisting of a small number of data points and not well distributed in radius. The case for NGC 3201 is encouraging in that Côté et al. (1995) obtained 857 radial velocities with median precision $\sim 1 \mathrm{~km} \mathrm{~s}^{-1}$ for 399 member giants to trace the velocity dispersion profile of this cluster out to 32 '. 1 . This has allowed nonparametric modeling of this cluster, and it was found that NGC 3201 has significantly more stars which are tightly bound (low energy) than King models predict (Gebhardt \& Fischer 1995).

\subsection{NGC 3201}

For NGC 3201, the tidal radius from the Wilson model is about twice as large as from the King model (see Table 1). The two RAVE extratidal stars are located $0.78^{\circ}$ and $0.88^{\circ}$ away in projection from the cluster center, well beyond the $0.47^{\circ}$ King tidal radius of the cluster (Harris 1996, 2010 edn.). Hence, either our results extend the velocity dispersion profile of this cluster out to $\sim 0.9^{\circ}$, inline with the Gebhardt \& Fischer (1995) findings and a Wilson tidal radius, or these RAVE stars are unbound and are therefore not part of the cluster's tidal radius. It is worth noting that NGC 3201 is thought to have experienced a recent impact with the Galactic disk (Vande Putte \& Cropper 2009), and this crossing could show stellar debris structure observed as clumpy structures (e.g., Chen \& Chen 2010). The extratidal RAVE stars may also be associated with just recently experienced disk-crossing shocks.

Curiously, despite the relatively rich number of RAVE cluster members found within the cluster tidal radius of NGC 3201, further than $0.9^{\circ}$, not one RAVE star with a radial velocity within $490 \mathrm{~km} \mathrm{~s}^{-1}$ and $500 \mathrm{~km} \mathrm{~s}^{-1}$ is found when searching 64206 RAVE stars within a $30^{\circ}$ radius of the cluster center (between the right ascension of $124.5^{\circ}$ and $184.5^{\circ}$ and the declination of $-76.5^{\circ}$ and $-16.5^{\circ}$ ). This suggests that if NGC 3201 has an extragalactic origin, no prominent, largescale stellar debris consistient with radial velocities between $490 \mathrm{~km} \mathrm{~s}^{-1}-500 \mathrm{~km} \mathrm{~s}^{-1}$ exists, such as would be perhaps expected if it were a satellite galaxy merging with the Milky Way. However, Côté et al. (1995) found a difference in the radial velocity of $1.22 \pm 0.25 \mathrm{~km} \mathrm{~s}^{-1}$ for stars on either side of the cluster, and although this could be interpreted as the cluster having internal rotation, it could also be that the radial velocity of the stellar population associated with NGC 3201 has a radial velocity gradient. There is one RAVE star outside the tidal radius of NGC 3201 with a radial velocity between $470 \mathrm{~km} \mathrm{~s}^{-1}-490 \mathrm{~km} \mathrm{~s}^{-1}$ and five RAVE stars with radial velocities between $500 \mathrm{~km} \mathrm{~s}^{-1}-520 \mathrm{~km} \mathrm{~s}^{-1}$, all located more than $20^{\circ}$ away from the cluster center. Therefore, the RAVE radial velocity data suggest that any potential tidal stream surrounding NGC 3201 is a weak feature. If this GC was accreted by the Milky Way much earlier, any debris could be quite diffuse and dynamically hot (e.g., Bullock \& Johnston 2005; Font et al. 2008). Detailed dynamical modeling of this cluster could address this further.

\subsection{NGC 1851}

There is increasing speculation that NGC 1851 is the result of a merger of two GCs that were both formed in a dwarf spheroidal galaxy, dragged to the center of the dSph by dynamical friction, and that now this dwarf spheroidal is being destroyed by Galactic tidal forces (e.g., van den Bergh 1996; Carretta et al. 2010). NGC 1851 has an unusually high central concentration of light, which can be explained if it was formed by the merging of two initially spherical star clusters (e.g., White 1978). Each cluster might have formed with a slightly different metallicity and with a different level of $\alpha$ elements, which would explain that the stars in NGC 1851 can be divided into two groups according to their metallicity, where both components show a moderate $\mathrm{Na}-\mathrm{O}$ anticorrelation (Carretta et al. 2010). Since the $\mathrm{Na}-\mathrm{O}$ (and the $\mathrm{C}-\mathrm{N}$ ) anticorrelations alone can be considered as the signature of multiple stellar populations, the two stellar groups in NGC 1851 both appear to exhibit the product of multiple stellar formation episodes.

That NGC 1851 was once part of a larger system, such as a dwarf spheroidal galaxy, is supported by its unexpected dispersion in heavy element abundances, which suggests enrichment by supernovae (Lee et al. 2009; Han et al. 2009). It is hard to 
understand how a GC with little or no dark matter and low binding energy could retain energetic supernovae ejecta, unless the GC we observe today were a remnant of an initially much more massive stellar system. Further, a diffuse stellar halo with a size of more than $500 \mathrm{pc}$ and a mass of $\sim 0.1$ per cent of the dynamical mass of NGC 1851 has been observed by Olszewski et al. (2009), and this peculiar feature can be explained by its formation in the central region of a defunct host dwarf galaxy (Bekki $\&$ Yong 2012). The different density distributions of metal-poor and metal-rich stars suggests that if NGC 1851 was formed from the merging of two clusters, this could not have occurred too long ago in the past (i.e., less than a few Gyr ago). This merging should have happened before the putative dwarf spheroidal dissolved, so it should still be possible to identify the remains of this ancestral, parent galaxy.

Given the state of published models, it is not possible to easily determine if the extratidal stars detected in RAVE are following the cluster's orbit, as is the case with Pal 5 and NGC 5466. The RAVE observations of candidate extratidal stars are also limited spatially, and we therefore cannot trace out discrete structures in common with photographic counts as in, for example, studies that find extensions of the cluster (Leon et al. 2000) or studies that show a smooth extended halo structure (Olszewski et al. 2009). It is, however, noteworthy that the extratidal candidate stars have a preferential direction toward a S-E extension, similar to the tails observed around NGC 1851 from Leon et al. (2000), see their Fig. 10.

To gain insight into where we should expect to find stars that were stripped off the cluster, we performed a simple (collisionless) $N$-body experiment ${ }^{2}$. We set a King model up with the structural properties of NGC 1851 as reported by Harris (1996) on an orbit around the Milky Way that would lead the cluster after $\sim 1$ Gyr to its present position and kinematics (taken from Harris 1996 and Dinescu et al. 1997). For the Galactic gravitational potential we use the mass model proposed by McMillan (2011). The resulting final angular positions of star particles are also shown in Fig. 2. As we start with the current structure of the cluster, which is then tidally stripped along its orbit, the final cluster in our model has slightly different properties than NGC 1851, but the simulation is still effective at identifying the rough location where the tidal debris would be observed.

The correlation between our NGC 1851 orbit and the candidate NGC 1851 extratidal RAVE stars is encouraging, and indicates a low probability that the majority of these stars are due to random fluctuations in the field. With suitable follow-up observations, they could be used as probes of a debris stream associated with NGC 1851.

Recently, a spectroscopic survey performed in the outskirts of NGC 1851 by Sollima et al. (2012) resulted in tentative evidence of a cold peak in the distribution of radial velocities at $\sim 180 \mathrm{~km} \mathrm{~s}^{-1}$. These stars had a location in the CMD compatible with a stream at a similar distance to this cluster, and if confirmed, would constitute a strong indication of the presence of a stream in the direction of NGC 1851. Therefore, we searched for an excess of RAVE stars with velocities of $\sim 180 \mathrm{~km} \mathrm{~s}^{-1}$ within 5 degrees of the cluster. Only one star with a radial velocity between $170 \mathrm{~km} \mathrm{~s}^{-1}$ and $190 \mathrm{~km} \mathrm{~s}^{-1}$ was identified with a similar location in the CMD as NGC 1851 , located $\sim 4.5^{\circ}$ from the cluster center. Hence, the RAVE data are not able to confirm a $180 \mathrm{~km} \mathrm{~s}^{-1}$ stream in the direction of NGC 1851, although given

\footnotetext{
2 The $N$-body integration was done using the code gyrfalcON (Dehnen 2002) which is publicly available in the $N$-body code frame work NEMO by Teuben (1995).
}

that the Sollima et al. (2012) feature was constituted mainly by main-sequence stars and the RAVE stars would only be able to probe the RGB and AGB stars, this is perhaps not surprising.

\subsection{NGC $6656-M 22$}

As discussed previously, the abundance spread in heavy elements in the stars of M 22 have caused much speculation that this cluster was previously a stellar nucleus formed in situ or through merging of two GCs with different chemical abundances. Despite being located in relatively close proximity to the Sun, $R_{\odot}=3.2 \mathrm{kpc}$, studies of this cluster are hampered by its location toward the crowded and heavily extincted Galactic bulge, making it difficult to disentangle cluster parameters, due to, for example, contamination from field stars and differential reddening (Monaco et al. 2004; Kunder et al. 2013).

The RAVE stars are limited in the direction of M22, yet we find a candidate extratidal star associated with M 22 based on $[\mathrm{Fe} / \mathrm{H}]$ metallicity, elemental abundances, and radial velocity, as well as its position on the RGB of M22. Similarly, within the ARGOS spectroscopic survey of the red clump stars of the Galactic bulge, Ness et al. (2013) find 11 stars with [Fe/H] abundances and radial velocities consistent with the GC M22. They find a mean distance spread of $1.8 \mathrm{kpc}$ and speculate that this distance spread is due in part to extended tidal streams associated with M 22. Unfortunately due to the unavailability of the ARGOS data and extremely limited data tables, we are unable to assess where these stars are spatially distributed and hence how far they are from the cluster center. The relative success of RAVE and ARGOS to kinematically select M 22 stars beyond its tidal radius suggests that a systematic spectroscopic search for M 22 extratidal stars would likely yield interesting results and perhaps a large enough sample to investigate the geometry of potential tidal tails associated with this cluster.

\subsection{Comments regarding $\omega$ Cen}

The GC $\omega$ Centauri (NGC 5129) is the largest GC known in the Milky Way galaxy with a radial velocity of $\sim 231 \mathrm{~km} \mathrm{~s}^{-1}$ and a large central velocity dispersion of $\sim 17 \mathrm{~km} \mathrm{~s}^{-1}$ (Harris 1996). The stars within this cluster show an unusually large star-to-star spread in iron that spans more than an order of magnitude, from $-2.2<[\mathrm{Fe} / \mathrm{H}]<-0.7$ (e.g., Johnson \& Pilachowski 2010). As already mentioned in the introduction, there has been evidence presented that this GC is the former nucleus of a dwarf galaxy (e.g., Lee et al. 1999; Bekki \& Freeman 2003; Majewski et al. 2012).

It is worth noting that there is a clear group of 14 stars with radial velocities $\sim 220-280 \mathrm{~km} \mathrm{~s}^{-1}$ as seen in the top, left panel of Fig. 1, and a group of 11 stars with radial velocities $\sim 220-280 \mathrm{~km} \mathrm{~s}^{-1}$ in the top, left panel of Fig. 2. The majority of these stars for which abundance determinations could be determined are also relatively metal-poor, as shown in the top, right panel of the same figures. These features are consistent with stars from the massive GC $\omega$ Cen, which has been shown to have a significant kinematically coherent "tidal debris" signature spanning $>60^{\circ}$ of Galactic longitude in the Grid Giant Star Survey (GGSS) radial velocity survey of giant stars located within $\sim 5 \mathrm{kpc}$ of the Sun (Majewski et al. 2012). This $\omega$ Cen signature is especially prominent at $l \sim 285^{\circ}$ (Majewski et al. 2012), and coincidentally, NGC 3201 is located at $l \sim 277^{\circ}$ and NGC 1851 is located at $l \sim 244^{\circ}$. Our radial velocity histograms covering the large areas surrounding NGC 1851 and NGC 3201 
therefore agree with the result from Majewski et al. (2012), that extended parts of the $\omega$ Cen tidal stream are contributing to giant stars in the inner Galaxy. It is also in agreement with the study of Wylie-de Boer et al. (2010), who find that a number of members of the Kapteyn group of nearby halo stars are probably remnants of tidal debris stripped from the parent galaxy of $\omega$ Cen, or from the cluster itself, during its merger with the Galaxy.

\section{Conclusions}

Stars selected from the extensive RAVE survey that can be associated with the GCs M22, NGC 1851 and NGC 3201 are presented, some reaching outside the King (1966) tidal radius of these clusters. For the kinematically peculiar cluster NGC 3201, we find cluster member stars that extend a few arc minutes from the edge of the cluster's radius and no further extratidal stars associated with NGC 3201. Given the relatively rich number of RAVE cluster members found within the cluster tidal radius of NGC 3201, the RAVE radial velocity data suggest that any potential tidal stream surrounding NGC 3201 would be a weak feature.

For M 22 and NGC 1851, two GCs with groups of stars with different $s$-element content, each group exhibiting their own Na-O, C-N anticorrelations, we find RAVE stars with radial velocities, $[\mathrm{Fe} / \mathrm{H}]$ abundances, elemental abundances, and positions on the CMD consistent with that of the cluster, but located at projected distances of $\sim 10$ degrees from the cluster center. Although the stellar proper motion errors of these stars are too large to use as an inclusion criteria, the UCAC4 proper motions are consistent with the small mean absolute proper motions of the corresponding clusters taking into account the errors. We conclude that these stars are promising extratidal candidates, suggesting an extragalactic origin for these clusters.

Since the tidal tails of GCs are primarily formed by the lowest-mass stars (e.g., Combes et al. 1999; Koch et al. 2004), it is challenging to study tidal tails of GCs using only bright stars. Therefore, it is difficult to use the RAVE results presented here to comment on the specifics of accreted GCs in the Milky Way, although if these extratidal stars are shown to be extended structures originating in the GC, then this provides spectroscopic evidence that accretion onto the early MW was significant, as predicted in the seminal work of Searle \& Zinn (1978). Following up with deep CCD studies in the location of the sky of these extratidal stars to search for the cluster's main-sequence, as in, for example, Carballo-Bello \& Martinez-Delgado (2010), would be a promising next step to confirm the association of these RAVE stars with the remnants of an accreted dwarf galaxy in the disk of the Milky Way.

Acknowledgements. The authors thank the reviewer for useful comments that helped improve and clarify this manuscript. This work was partially supported by PRIN-INAF 2011 "Tracing the formation and evolution of the Galactic halo with VST" (P.I.: Marconi) and by PRIN-MIUR (2010LY5N2T) "Chemical and dynamical evolution of the Milky Way and Local Group galaxies" (P.I.: Matteucci). Funding for RAVE has been provided by: the Australian Astronomical Observatory; the Leibniz-Institut fuer Astrophysik Potsdam (AIP); the Australian National University; the Australian Research Council; the French National Research Agency; the German Research Foundation (SPP 1177 and SFB 881); the European Research Council (ERC-StG 240271 Galactica); the Istituto Nazionale di Astrofisica at Padova; The Johns Hopkins University; the National Science Foundation of the USA (AST-0908326); the W. M. Keck foundation; the Macquarie University; the Netherlands Research School for Astronomy; the Natural Sciences and Engineering Research Council of Canada; the Slovenian Research Agency; the Swiss National Science Foundation; the Science \& Technology Facilities Council of the UK; Opticon; Strasbourg Observatory; and the Universities of Groningen, Heidelberg and Sydney. The RAVE web site is at http://www. rave-survey.org

\section{References}

Abadi, M. G., Navarro, J. F., \& Steinmetz, M. 2006, MNRAS, 365, 747 Alves-Brito, A., Yong, D., Meléndez, J., Vásquez, S., \& Karakas, A. I. 2012, A\&A, 540, A3

Bedin, L. R., Piotto, G., \& Anderson, J., et al. 2004, ApJ, 605, L125

Belokurov, V., Evans, N. W., Irwin, M. J., Hewett, P. C., \& Wilkinson, M. I. 2006, ApJ, 637, 29

Bekki, K., \& Freeman, K. C. 2003, MNRAS, 346, 11

Bekki, K., \& Yong, D. 2012, MNRAS, 419, 2063

Binney, J., \& Merrifield, M. 1998, Galactic Astronomy (Princeton: Princeton Univ. Press), 307

Binney, J., Burnett, B., Kordopatis, G., et al. 2014, MNRAS, 439, 1231

Boeche, C., Siebert, A., Williams, M., et al. 2011, AJ, 142, 193

Bullock, J. S., \& Johnston, K. V. 2005, ApJ, 635, 931

Carballo-Bello, J. A., \& Martinez-Delgado, D. 2010, in Highlights of Spanish Astrophysics V, eds. J. M. Diego, L. J. Goicoechea, J. I. González-Serrano, \& J. Gorgas (Berlin: Springer-Verlag), 383

Carretta, E., Bragaglia, A., Gratton, R., \& Lucatello, S. 2009, A\&A, 505, 139

Carretta, E., Gratton, R. G., Lucatello, S., et al. 2010, ApJ, 722, 1

Carretta, E., Lucatello, S., Gratton, R. G., et al. 2011, A\&A, 533, A69

Carretta, E., D’Orazi, V., Gratton, R. G., \& Lucatello, S. 2012, A\&A, 543, A117

Chen, C. W., \& Chen, W. P. 2010, ApJ, 721, 1790

Crnojević, D., Grebel, E. K., \& Koch, A. 2010, A\&A, 516, A85

Cohen, J. G., Kirby, E. N., Simon, J. D., \& Geha, M. 2010, ApJ, 725, 288

Combes, F., Leon, S., \& Meylan, G. 1999, A\&A, 352, 149

Côté, P., Welch, D. L., Fischer, P., et al. 1994, ApJS, 90, 83

Côté, P., Welch, D. L., Fischer, P., \& Gebhardt, K. 1995, ApJ, 454, 788

Da Costa, G. S., \& Armandroff, T. E. 1995, AJ, 109, 2533

Da Costa, G. S., Held, E. V., Saviane, I., \& Gullieuszik, M. 2009, ApJ, 705, 1481

Dehnen, W. 2002, ASPC, 275, 105

Di Cecco, A. Zocchi, A., Varrie, A. L., et al. 2013, AJ, 145, 103

Di Criscienzo, M., D'Antona, F., \& Milone, A. P., et al. 2011, MNRAS, 414, 3381

Dinescu, D. I., Girard, T. M., van Altena, W. F., Méndez, R., \& López, C. E. 1997, AJ, 114, 1014

Ferraro, F. R., Dalessandro, E., \& Mucciarelli, A., et al. 2009, Nature, 462, 483

Font, A. S., Johnston, K. V., Ferguson, A. M. N., et al. 2008, ApJ, 673, 215

Forbes, D. A., \& Bridges, T. 2010, MNRAS, 404, 1203

Freeman, K. C. 1993, in The Globular Clusters-Galaxy Connection, eds. G. Smith, \& J. Brodie (San Francisco: ASP), ASP Conf. Ser., 48, 608

Freeman, K. C., \& Rodgers, A. W. 1975, ApJ, 201, 71

Gebhardt, K., \& Fischer, P. 1995, AJ, 109, 290

Geyer, M. P., \& Burkert, A. 2001, MNRAS, 323, 988

Gierz, M., \& Heggie, D. C. 1997, MNRAS, 286, 709

Gnedin, O. Y., \& Ostriker, J. P. 1997, ApJ, 474, 223

Gonzalez, G., \& Wallerstein, G. 1998, AJ, 116, 765

Gratton, R. G., Sneden, C., \& Carretta, E. 2004, ARA\&A, 42, 385

Gratton, R. G., Carretta, E., \& Bragaglia, A. 2012a, A\&ARv, 20, 50

Gratton, R. G., Villanova, S., Lucatello, S., et al. 2012b, A\&A, 544, A12

Gratton, R. G., Lucatello, S., Carretta, E., et al. 2012c, A\&A, 539, A19

Gratton, R. G., Lucatello, S., Sollima, A., et al. 2014, A\&A, 563, A13

Grillmair, C. J. 2009, ApJ, 693, 1118

Grillmair, C. J. 2011, ApJ, 738, 98

Grillmair, C. J., \& Dionatos, O. 2006, ApJ, 641, 37

Grillmair, C. J., \& Johnson, R. 2006, ApJ, 639, 17

Grillmair, C. J., Freeman, K. G., Irwin, M., \& Quinn, P. J. 1995, AJ, 109, 2553

Grillmair, C. J., Ajhar E. A., Faber, S. M., et al. 1996, AJ, 111, 2293

Grillmair, C. J., Cutri, R., Masci, F. J., et al. 2013, ApJ, 769, 23

Groenewegen, M. A. T., Udalski, A., \& Bono, G. 2008, A\&A, 81, 441

Harris, W. E. 1996, AJ, 112, 1487

Han, S.-I., Lee, Y.-W., \& Joo, S.-J., et al. 2009, ApJ, 707, L190

Holland, S., Fahlman, G. G., \& Richer, H. B. 1997, AJ, 114, 1488

Johnson, C. I., \& Pilachowski, C. A. 2010, ApJ, 722, 1373

Jordi, K., \& Grebel, E. K. 2010, A\&A, 522, A71

King, I. R. 1966, AJ, 71, 64

Koch, A., Grebel, E. K., Odenkirchen, M., Martínez-Delgado, D., \& Caldwell, J. A. R. 2004, AJ, 128, 2274

Kordopatis, G., Gilmore, G., Steinmetz, M., et al. 2013, AJ, 146, 134

Kroupa, P. 2001, MNRAS, 322, 231

Kunder, A., Stetson, P. B., Cassisi, S., et al. 2013, AJ, 146, 119

Layden, A. C., \& Sarajedini, A. 1997, ApJ, 486, L107

Layden, A. C., \& Sarajedini, A. 2000, AJ, 119, 1760

Leaman, R., VandenBerg, D. A., \& Mendel, J. T. 2013, MNRAS, 436, 122

Lee, Y.-W., Joo, J.-M., \& Sohn, Y.-J., et al. 1999, Nature, 402, 55

Lee, K. H., Lee, H. M., Fahlman, G. G., \& Lee, M. G. 2003, AJ, 126, 815

Lee, K. H., Lee, H. M., Fahlman, G. G., \& Sung, H. 2004, AJ, 128, 2838

Lee, J-W., Lee, J., Kang, Y.-W., et al. 2009, ApJ, 695, L78 
Lehmann, I., \& Scholz, R.-D. 1997, A\&A, 320, 776

Leon, S., Bergond, G., \& Vallenari, A. 1999, A\&A, 344, 450

Leon, S., Meylan, G., \& Combes, F. 2000, A\&A, 359, 907

Mackey, A. D., \& Gilmore, G. F. 2004, MNRAS, 355, 504

Majewski, S. R., Nidever, D. L., Smith, V. V., et al. 2012, ApJ, 747, 37

Marín-Franch, A., Aparicio, A., Piotto, G., et al. ApJ, 2009, 694, 1498

Marino, A. F., Milone, A. P., Piotto, G., et al. 2009, A\&A, 505, 1099

Marino, A. F., Sneden, C., \& Kraft, R. P., et al. 2011, A\&A, 532, A8

Marino, A. F., Milone, A. P., Sneden, C., et al. 2012, A\&A, 541, A15

Martell, S. L., \& Grebel, E. K. 2010, A\&A, 519, A14

Martell, S. L., Smolinski, J. P., Beers, T. C., \& Grebel, E. K. 2011, A\&A, 534, A136

Matsunaga, N., Kawadu, T., Nishiyama, S., et al. 2009, MNRAS, 399, 1709

McLaughlin, D. E., \& van der Marel, R. P. 2005, ApJS, 161, 304

McMillan, P. J. 2011, MNRAS, 414, 2446

Meylan, G., \& Heggie, D. C. 1996, A\&ARv, 8, 1

Milone, A. P., Bedin, L. R., Piotto, G., et al. 2008, ApJ, 673, 241

Milone, A. P., Stetson, P. B., Piotto, G., et al. 2009, A\&A, 503, 755

Milone, A. P., Piotto, G., Bedin, L. R., et al. 2012, ApJ, 744, 58

Monaco, L., Pancino, E., Ferraro, F. R., \& Bellazzini, M. 2004, MNRAS, 349, 1278

Montuori, M., Capuzzo-Dolcetta, R., Di Matteo, P., Lepinette, A., \& Miocchi, P. 2007, ApJ, 659, 1212

Munari, U., Siviero, A., Bienayme, O., et al. 2009, A\&A, 503, 511

Muñoz, C., Geisler, D., \& Villanova, S. 2013, MNRAS, 433, 2006

Murali, C., \& Dubinski, J. 1999, AJ, 118, 911

Ness, M., Freeman, K., Athanassoula, E., et al. 2013, MNRAS, 430, 936

Norris, J., \& Freeman, K. C. 1983, ApJ, 266, 130

Olszewski, E. W., Saha, A., Knezek, P., et al. 2009, AJ, 138, 1570

Odenkirchen, M., Grebel, E. K., Rockosi, C. M., et al. 2001, ApJ, 548, 165

Odenkirchen, M., Grebel, E. K., \& Dehnen, W., et al. 2003, AJ, 126, 2385

Odenkirchen, M., Grebel, E. K., Kayser, A., Rix, H.-W., \& Dehnen, W. 2009, AJ, 137, 3378

Peñarrubia, J., Walker M. G., \& Gilmore, G. 2009, MNRAS, 399, 1275

Richer, H. B., Heyl, J., Anderson, J., et al. 2013, ApJ, 771, 15
Rodgers, A. W., \& Paltoglou, G. 1984, ApJ, 283, L5

Romano, D., Matteucci, F., Tosi, M., et al. 2006, MNRAS, 376, 405

Salaris, M., \& Weiss, A. 2002, A\&A, 388, 492

Sarajedini, A., \& Layden, A. C. 1995, 109, 1086

Searle, L., \& Zinn, R. 1978, ApJ, 225, 357

Siegel, M. H., Dotter, A., \& Majewski, S. R., et al. 2007, ApJ, 667, L57

Simmerer, J., Ivans, I. I., Filler, D., et al. 2013, ApJ, 764, 7

Sohn, Y.-J., Park, J.-H., Rey, S.-C., et al. 2003, AJ, 126, 803

Sollima, A., Gratton, R. G., Carballo-Bello, J. A., et al. 2012, MNRAS, 426, 1137

Steinmetz, M., Zwitter, T., Siebert, A., et al. 2006, AJ, 132, 1645

Testa, V., Zaggia, S. R., Andreon, G. L., et al. 2000, A\&A, 356, 127

Teuben, P. 1995, ASP Conf., 77, 398

Unavane, M., Wyse, R. F. G., \& Gilmore, G. 1996, MNRAS, 278, 727

van den Bergh, S. 1993, AJ, 105, 971

van den Bergh, S. 1996, ApJ, 471, 31

VandenBerg, D. A., Brogaard, K., Leaman, R., \& Casagrande, L. 2013, ApJ, 775,134

Vande Putte, D., \& Cropper, M. 2009, MNRAS, 392, 113

Villanova, S., Geisler, D., \& Piotto, G. 2010, ApJ, 722, 18

Williams, M. E. K., Steinmetz, M., Sharma, S., et al. 2011, ApJ, 728, 102

Willett, B. A., Newberg, H. J., Zhang, H., Yanny, B., \& Beers, T. 2009, ApJ, 697, 207

Wilson, C. P. 1975 , AJ, 80, 175

White, S. D. M. 1978, MNRAS, 184, 185

Wright, E., Eisenhardt, P. R. M., \& Mainzer, A. K., et al. 2010, AJ, 140, 1868 Wylie-de Boer, E., Freeman, K., \& Williams, M. E. K. 2010, AJ, 139, 636 Wylie-de Boer, E., Freeman, K., Williams, M. E. K., et al. 2012, ApJ, 755, 35 Yong, D., \& Grundahl, F. 2008, ApJ, 672, 29

York, D. G., Adelman, J., Anderson, J. E., Jr., et al. 2000, AJ, 120, 1579

Zinnecker, H., Keable, C. J., Dunlop, J. S., Cannon, R. D., \& Griffiths, W. K. 1988, in The Harlow Shapley Symposium on Globular Cluster Systems in Galaxies, eds. J. E. Grindlay, \& A. G. D. Philip, The Nuclei of Nucleated Dwarf Elliptical Galaxies - are they Globular Clusters? IAU Symp., 126, 603 Zocchi, A., Bertin, G., \& Varri, A. L. 2012, A\&A, 539, A65 